\title{
Intoxicación aguda por cocaína. A propósito de un caso
}

\author{
M. SOLANO REMÍREZ, J. P. VELILLA ALCUBILLA, M. T. ÁLVAREZ FRÍAS \\ Servicio de Medicina Interna. Hospital de Navarra. Pamplona
}

ACUTE INTOXICATION DUE TO COCAINE. REVIEW OF ONE CASE

\begin{abstract}
RESUMEN
La cocaína es una droga con amplia distribución y consumo en el mundo desarrollado. Su consumo se extendió en los decenios 1980-1990 sobre todo. Son conocidos sus efectos cardiovasculares nocivos tanto en su consumo crónico o/y en sobredosis, debido a su potente efecto vasoconstrictor secundario a liberación hormonas catacolaminérgicas como por su inhibición de la recaptación de noradrenalina. Tanto la insuficiencia renal, sobre todo secundaria a rabdomiolisis, como los trastornos cardiovasculares agudos constituyen los efectos tóxicos más frecuentes y conocidos, que pueden llegar a ser mortales. Es por ello que describimos un caso en el mismo paciente donde concurren ambos eventos patológicos, para describir y actualizar los efectos nocivos del abuso de cocaína y su tratamiento.
\end{abstract}

PALABRAS CLAVE: Intoxicación cocaína. Rabdomiolisis. Insuficiencia renal aguda. Edema agudo de pulmón.

\begin{abstract}
Cocaine is a drug with distribution and consumption throughout the world. Its consumption extended mainly in the decades 1980-1990. The drugs produces cardiovascular effects following chronic administration and overdose, due to their potent vasoconstrictor effect secondary to the increase of synaptic concentrations of the monamine neurotransmitter dopamine, norepinphrine, and serotonin by binding to transporter proteins and blocking reuptake. Renal insufficiency due to rhabdomyolysis, and cardiovascular dysfunction have been reported following cocaine abuse, and may be fatal. We report a patient who developed renal insufficincy due to rhabdomyolysis and a cardiovascular disorder associated with cocaine abuse. We reviewew the disorders induced by cocaine abuse and their treatment.
\end{abstract}

KEY WORDS: Cocaine intoxication. Rhabdomyolysis. Acute renal failure. Acute pulmonary edema.

Solano Remírez, M, Velilla Alcubilla JP, Álvarez Frías MT. Intoxicación aguda por cocaína. A propósito de un caso. An Med Interna (Madrid) 2006; 23: 31-33.

\section{INTRODUCCIÓN}

El consumo de cocaína en sus diferentes formas, crónica o en sobredosis, puede producir una potente acción simpaticomimética debida a sus efectos en la liberación de dopamina y catecolaminas, así como por la inhibición de la recaptación de noradrenalina, lo que conlleva a eventos cardiovasculares y renales que pueden llegar a ser mortales. El mecanismo etiopatogénico de su acción sigue sin ser conocido con exactitud. Así mismo puede producir inhibición del efecto vasodilatador del óxido nítrico y activación directa de la contracción del músculo liso vascular calcio-mediado. A nivel muscular se puede producir un efecto vasoconstrictor masivo que puede llevar a isquemia aguda que se traduciría en rabdomiolisis con importante elevación de la creatin-kinasa y conllevar a insuficiencia renal aguda. Por todo ello pueden producirse situaciones de emergencia hipertensiva, accidentes cardiovasculares a nivel cerebral y/o miocardio, renal y muscular. Uno de los órganos afectados es el riñón que a menudo sufre insuficien- cia renal aguda por diferentes mecanismos (nefroangioesclerosis, infarto renal, etc.) siendo lo más frecuentemente secundario a rabdomiolisis.

\section{CASO APORTADO}

Se trata de un varón de 33 con antecedentes personales de exADVP en tratamiento sustitutivo con Metadona, consumidor habitual de cocaína y cannabis, diagnosticado de HVC (con biopsia hepática realizada), herida por asta de vaca brava EII, intervenido de necrosis avascular de cadera derecha, fractura de hombro y muñeca derecha. Como tratamiento previo llevaba Tranxilium $50 \mathrm{mg} 1 / 2$ comprimido cada 8 horas. Ingresa tras ser encontrado en domicilio por familiares en situación de disminución del nivel de conciencia con restos de vómito alimentario y edema en extremidad superior izquierda; refería haber consumido previamente cocaína vía intranasal y consumo de alcohol concomitante las horas previas. Así mismo refería dolor e inflamación en ESI. Presentaba, a la exploración física, febrícula de $37,5^{\circ} \mathrm{C}$, TA $120 / 90$, consciente orientado con bajo 
nivel de conciencia sin focalidad neurológica, edema y dolor con edema en ESI. Al realizar primera valoración realiza escasa emisión de orina colúrica. En el ECG un ritmo sinusal a 105 lpm. La radiografía de tórax ligera cardiomegalia. En la analítica de sangre CPK 33.255 U/L (normal 10-80), ASAT 1421 U/L (normal 0-18), ALAT $596 \mathrm{U} / \mathrm{L}$ (normal 0-22), calcio 9,4 mg/dL, amilasa $396 \mathrm{U} / \mathrm{L}$ (normal $36-128$ ), urea $135 \mathrm{mg} / \mathrm{dL}$ (normal 10-50) y creatinina $11,8 \mathrm{mg} / \mathrm{dL}$ (normal 0,6-1,2). El sedimento urinario fue normal. Se realizó determinación de tóxicos en orina que fue positivo para cannabinoides, metadona, benzodiacepinas y cocaína. Los niveles de etanol en sangre en cantidad no mensurable. Se comenta con Servicio de Nefrología que realiza varias sesiones de hemodiálisis hasta entrar en fase de poliuria y recuperación de la función renal (creatinina de $1.6 \mathrm{mg} / \mathrm{dL}$ ), mejorando los parámetros enzimas hepáticos (ASAT 47 U/L, ALAT 95 U/L) y con CPK de 80 U/L. El paciente es dado de alta. A los 6 días es traído de nuevo por familiares por presentar estado confusional y agitación psicomotriz con fiebre de $39,5^{\circ} \mathrm{C}$. Refería haber fumado las horas previas $50 \mathrm{gr}$ de cocaína mezclada con amoniaco. Presentaba fiebre de $40{ }^{\circ} \mathrm{C}$, taquipnea a $47 \mathrm{rpm}$ y agitación psicomotriz, a la auscultación pulmonar crepitantes bilaterales; la auscultación cardiaca reflejaba taquicardia sin soplos añadidos; no ingurgitación yugular; además se objetivó hipoxemia parcial moderada $\left(\mathrm{pO}_{2}\right.$ $76 \mathrm{mmHg}, \mathrm{pCO}_{2} 40 \mathrm{mmHg}, \mathrm{SatO}_{2} 80 \%, \mathrm{pH} \mathrm{7,4}$, exceso base 0 $\mathrm{mmol} / \mathrm{L}$. El ECG estaba en ritmo sinusal a $108 \mathrm{lpm}$. La radiografía de tórax evidenciaba un infiltrado intersticial bilateral con leve cardiomegalia. En la analítica de sangre destacaba leucocitos 13.100 (normal 3,5-10,5), ALAT $221 \mathrm{U} / \mathrm{L}$ (normal 0-22), ASAT $338 \mathrm{U} / \mathrm{L}$ (normal 0-18), creatinina 2,9 mg/dL (normal 0,6-1,2), calcio 8,4 $\mathrm{mg} / \mathrm{dL}$, urea $88 \mathrm{mg} / \mathrm{dL}$ (normal 10-50), CPK $776 \mathrm{U} / \mathrm{L}$ (normal 1080). Así mismo en orina se determinó positividad para cannabinoides, metadona y cocaína. Los niveles de etanol en sangre fueron 0,1 $\mathrm{mg} / \mathrm{L}$ (normal 0-0,5). Se inició tratamiento con sueroterapia, antibioticoterapia de amplio expectro empírica y oxigenoterapia en ventimask a 10 L (no precisó ventilación mecánica), con evolución favorable, precisando sedación mientras persistió inicialmente la agitación psicomotriz. Al alta la radiografía tórax mostraba practica resolución infiltrado bilateral, en la analítica creatinina $0,9 \mathrm{mg} / \mathrm{dL}$, ASAT 41 U/L, ALAT 149 U/L, leucocitos 7.000, urea 32.

\section{DISCUSIÓN}

El consumo de cocaína se popularizó en la década de 1980 en Estados Unidos, de tal forma que en la década de 1990 hasta el $11 \%$ de los estadounidenses mayores de 12 años había consumido alguna vez cocaína en alguna de sus formas. En España sigue siendo en la actualidad la droga más consumida. Los primeros datos arqueológicos donde se observó el consumo humano de la cocaína se remonta a 3.000 a.c. La cocaína es un alcaloide derivado de la planta de coca, extendida y originaria de los países de América del Sur. La cocaína constituye el 1\% del peso de la hoja de coca. La mayoría de la coca se trasforma a su forma en polvo (clorhidrato de cocaína), la cual es soluble en agua y puede absorberse por vía intranasal o inyectada.. Debida a su alto punto de fusión el clorhidrato se descompone al ser quemado, por lo que para ser consumida fumada debe ser trasformada en su forma alcaloide, por lo que se disuelve en agua mezclada con sosa y se calienta, produciéndose precipitados ; algunos emplean amoniaco $(1,2)$. El clorhidrato de cocaína en polvo para inhalar se suele mezclar con manitol o lactosa para cortar. La cocaína fumada tiene una absorción muy rápida (6-8 segundos) a la circulación cerebral con un mayor volumen de distribución que la consumida por vía intranasal (ya que esta tiene limitada su absorción por el efecto vasoconstrictor a nivel nasal). La biodisponibilidad es variable, entre el 20-60\%, siendo más elevada en la forma fumada. La vida media de la cocaína en sangre es de aproximadamente 1 hora, siendo la eliminación en orina del $5 \%$ de la cocaína consumida pudiéndose determinar su presencia en las primeras 24 horas independientemente de la forma administrada. El riñón es unos de los órganos más afectados por el consumo de cocaína. La esterificación en plasma y en hígado de la cocaína produce varios metabolitos con una vida media de 4-6 horas, de los cuales, uno de ellos se puede encontrar en orina en casi 50-100 mayor concentración que la cocaína, siendo este el empleado para la prueba de determinación en orina. Se ha postulado que los pacientes con deficiencia de una de las esterasas en plasma encargadas de la metabolización de la cocaína (pseudocolinesterasa), pueden ser más sensibles a la toxicidad producida por el consumo de cocaína. Se han descrito una variada gama de complicaciones asociadas al uso de cocaína (Tabla I). El método o forma de consumo afecta al tipo de complicación, aunque el conocimiento de la toxicidad de cocaína se deriva de casos individuales y de algunas pequeñas series de pacientes observados. La cocaína produce contracción directa del músculo liso vascular (este depende de la actividad metabólica endotelial del calcio y por otra parte de la vasodilatación medida por el óxido nítrico); además posee efecto vasoconstrictor mediado por la liberación de dopamina y catecolaminas y por la inhibición de la recaptación de noradrenalina (3). El riñón es uno de los órganos afectados por la intoxicación de cocaína. Se han descrito variso efectos fisiopatológicos (inducción de aterogénesis, cambios

$\begin{array}{lc} & \text { TABLA I } \\ \text { Cardiacas } & \text { Pulmonares } \\ \text { Dolor torácico } & \text { Neumotórax } \\ \text { Infarto de miocardio } & \text { Neumomediastino } \\ \text { Arritmias } & \text { Neumopericardio } \\ \text { Miocardiopatía } & \text { Edema pulmonar } \\ \text { Miocarditis } & \text { Exacerbaciones de asma } \\ & \text { Bronquiolitis obliterante } \\ \text { Endocrinas } & \text { Hemorragia pulmonar } \\ \text { Hiperprolactinemia } & \\ & \text { Psiquiátricas } \\ \text { Gastrointestinales } & \text { Ansiedad } \\ \text { Isquemia intestinal } & \text { Depresion } \\ \text { Perforaciones gastrointestinales } & \text { Delirios. Paranoia } \\ \text { Colitis } & \text { Psicosis } \\ & \text { Suicidios } \\ \text { ORL y Oftalmológicas } & \\ \text { Ulceraciones gingivales } & \text { Renales } \\ \text { Erosiones dentales } & \text { Rabdomiolisis } \\ \text { Queratitis } & \text { Insuficiencia renal aguda } \\ \text { Defectos corneales epiteliales } & \text { Nefritis intersticial aguda } \\ \text { Rinitis crónica } & \text { Nefropatía tubular aguda } \\ \text { Perforación septo nasal } & \\ \text { Granuloma de la linea media } & \text { Obstétricas } \\ \text { Alteraciones olfatorias } & \text { Desprendimiento placenta } \\ \text { Neuropatía óptica } & \text { Prematuridad } \\ \text { Sinusitis osteolítica } & \text { Microcefalia } \\ \text { Neurológicas ACVA } & \text { Bajo peso } \\ \text { Crisis epilépticas } & \\ \text { Atrofia cerebral } & \text { Otras } \\ \text { Vasculitis cerebral } & \text { Disfunción sexual } \\ \text { Cefaleas/migrañas } & \text { Hiperpirexia } \\ & \text { Muerte repentina } \\ & \end{array}$


hemodinámicos y alteración en la síntexis de la matriz mesangial). La insuficiencia renal secundaria a rabdomiolisis es la complicación renal más frecuente del consumo de cocaína $(4,13,14)$. Los pacientes suelen tener síntomas leves de tipo neuromuscular. En estudios realizados en pacientes con efectos secundarios del consumo de cocaína hasta en un $24 \%$ se encontraron síntomas relacionados con la rabdomiolisis $(15,16)$. La rabdomiolisis viene definida por la elevación de más de 5 veces los valores séricos normales de la enzima creatín-kinasa. En una de las series más extensa de pacientes con rabdomiolisis secundaria al consumo de cocaína aproximadamente 1/3 asociaban insuficiencia renal aguda, de los cuales la mitad aproximadamente falleció. La tasa media de creatín-kinasa sérica, en esta serie, era de $12.000 \mathrm{U} / \mathrm{L}$, siendo de $28.000 \mathrm{U} / \mathrm{L}$ la de los pacientes que fallecieron por fallo renal agudo (17). Se ha determinado que uno de los factores de mayor severidad de la rabdomiolisis la constituía era el nivel sérico bajo del calcio. En los casos de rabdomiolisis asociado a fallo renal agudo se asoció a citolisis hepática y, ocasionalmente, a coagulación intravascular diseminada (6). El mecanismo etiopatogénico de la rabdomiolisis es, aún en la actualidad, confuso pudiendo asociarse a hipertermia en ocasiones (4,7). Las complicaciones pulmonares descritas se han asociado más frecuentemente con el consumo de cocaína fumada, siendo las más frecuentes, el barotrauma (neumotórax, neumomedistino, neumopericardio) $(8,9,11)$. Ocasionalmente se han descrito casos de edema pulmonar no cardiogénico (5). El mecanismo subyacente del edema agudo pulmonar no está bien determinado, aunque se han barajado como posibles causas la disfunción ventricular izquierda transitoria o la alteración de la permeabilidad capilar pulmonar (esta con mayor probabilidad, ya que en algunos pacientes se realizó lavado broncoalveolar, encontrándose niveles elevados de proteína) $(2,12,18)$. En la radiografía de estos pacientes la silueta cardiaca suele ser de tamaño y características radiológicas normales. Se descrito exar- cebaciones con el consumo de cocaína en pacientes con antecedentes de asma, ya que la cocaína tanto inhalada como fumada produce irritabilidad bronquial inespecífica. En otras series se relacionó el consumo de cocaína con hemorragia pulmonar (se encontraron, en el lavado broncoalveolar, macrófagos cargados de hemosiderina) y bronquiolitis $(9,10)$. En pruebas funcionales respiratorias realizadas a pacientes consumidores crónicos de cocaína se observó disminución de la difusión del monóxido de carbono, pero no se objetivaron alteraciones en las espirometrías realizadas a estos pacientes.

En nuestro caso en el primer ingreso presentó insuficiencia renal aguda por rabdomiolisis masiva sin datos de hipotensión comportándose como una necrosis tubular aguda sin proteinuría acompañado de citólisis hepática importante con fallo hepato-renal agudo que precisó varias sesiones de hemodiálisis; presentó evolución favorable y recuperación de la función renal además de normalización de enzimas hepáticas, a pesar de los antecedentes de hepatopatía crónica pos HVC, El calcio se encontraba en valores séricos dentro de la normalidad. En el segundo ingreso presento episodio compatible con edema agudo de pulmón , además de hiperpirexia, debiéndose hacer diagnóstico diferencial entre edema de perfil cardiológico, reagudización asmática (dado los antecedentes), edema no cardiogénico, hemorragia pulmonar y bronquiolitis obliterante. Dado la exploración física (no existía hiperreactividad bronquial, datos de arritmias ni HTA, ni datos clínicos de insuficiencia cardiaca -ingurgitación yugular, edemas, etc.-) y los datos radiológicos (ausencia de cardiomegalia clara ni engrosamiento hiliar) además de la favorable evolución con medidas preventivas y diuréticos, nos inclinamos por edema de pulmón no cardiogénico probablemente por aumento de presión alveolar capilar, no pudiendose descartar hemorragia pulmonar ni bronquiolitis ya que no se realizó lavado broncoalveolar confirmatorio de estas dos últimas entidades.

\section{Bibliografía}

1. Baldwin GC, Choi R, Roth D, Shay AH, Kleerup EC, MS Simmons, Taskin DP. Evidence of Chronic Damage to the Pulmonary Microcirculation in habitual users of alkaloidal ("crack") cocaine. Chest 2002; 121: 1231-1238.

2. Kleerup EC, Koyal SN, Marques-Magallanes JA, Goldman MD, Taskhin DP. Chronic and acute effects of "crack" cocaine on diffsing capacity, membrane difufusion, and pulmonary capillary blood volume in the lung. Chest 2002; 122: 629-638.

3. Jacobsen TN, Grayburn PA, Snyder II RW, Hansen J, Chavoshan B, Landau C, et al. Effects of intranasal cocaine on sympathetic nerve discharge in humans. J Clin Invest 1997; 99: 628-634.

4. Vallina Álvarez E, Suárez Arias R, García Díez A, Arribas Castrillo JM. Síndrome de rabdomiolisis e hipertermia secundario al consumo de cocaína y/o éxtasis. Comunicación de dos nuevos casos y revisión del problema. An Med Interna (Madrid) 2002; 19: 2; 85-88.

5. Cucco RA, Yoo OH, Cregler L, Chang JC. Nonfatal pulmonary edema after "freebase" cocaine smoking. Am Rev Respir Dis 1987; 136: 17981.

6. Campos Franco J, Martínez Rey C, Pérez Becerra E, González Quintela A. Fallo hepático fulminante asociado al uso de cocaína. An Med Interna (Madrid) 2002; 19: 365-367.

7. Rodríguez Jornet A, Andreu Navarro FJ, de Mendoza Asensi D, Carvajal Díaz A, Sala Rodó M, Cervantes García M, et al. Hipertensión arterial maligna e insuficiencia renal aguda por consumo de cocaína. Nefrología 2000; 20: 501-509

8. García Castaño J, Muiño Miguez A, González López-Cobos C, Gilsanz Fernández C. Complicaciones pulmonares y cocaína. A propósito de un

nuevo caso de neumomediastino. An Med Interna (Madrid) 1994; 11 : 519.

9. Murray RF, Albin RJ, Mergner W, Criner GJ. Diffuse alveolar hemorrhage temporarlly related to cocaine smoking. Chest. 1988; 93: 427-9.

10. Patel RC, Dutta D, Schonfeld SA. Free-base cocaine use associated with bronchiolitis obliterans organizing pneumonia. An Med Interna (Madrid) 1987; 107: 186-7.

11. Forrester JM, Steele AW, Waldron JA, Parsons PE. Crack lung: an acute pulmonary syndrome with a spectrum of clinical and histopathologic findings. Am Rev Respir Dis 1990; 142: 462-7.

12. Singh PP, Dimich L, Shamsi A. Intraoperative pulmonary oedema in a young cocaine smoker. Can Anaesth 1994; 41: 961-964.

13. Herzlich BC, Arsura EL, Pagala M, Grob D. Rhabdomyolysis related to cocaine abuse. Ann Intern Med 1988; 109: 335-6.

14. Amoedo Ml, Craver L, Marco MP, Fernández E. Cocaine-induced acute renal failure without rahbdomyolisis. Nephrol Dial Transplant 1999; 14: 2970-1

15. Welch RD, Todd K, Krause GS. Incidence of cocaine-associated rhabdomyolysis. Ann Emerg Med 1991; 20: 154-7.

16. Di paolo N, Fineschi V, Di Paolo M, Wetly CV, Garosi G, Del Vecchio MT, et al. Kidney vascular damage and cocaine. Clin Nephrol 1997; 47: 298-30.

17. Thakur V, Godley C, Weed S, Cook ME, Hoffman E. Cocaine-associates accelerated hypertension and renal failure. Am J Med Sci 1996; 312: $295-8$

18. Lange RA, Hillis LD. Cardiovascular complications of cocaine use. N Engl Med 2001; 345: 351-8. 